\title{
Freshwater crayfish in Bosnia and Herzegovina: the first report on their distribution
}

\author{
S. Trožić-Borovac ${ }^{(1)}$
}

Received January 14, 2011

Revised April 30, 2011

Accepted May 20, 2011

\section{ABSTRACT}

Key-words:

crayfish,

diversity,

Bosnia

and Herzegovina
The aim of this research paper is to present available data on the distribution of freshwater crayfish in Bosnia and Herzegovina, including data from the literature and those of our own investigations. According to historical data, the existence of four native European species were reported within the territory of Bosnia and Herzegovina: Astacus astacus (Linnaeus, 1758), A. leptodactylus (Eschscholtz, 1823), Austropotamobius torrentium (Schrank, 1803) and A. pallipes (Lereboullet, 1858). Our recent research has confirmed the presence of these four species. A. torrentium has been found in the tributaries of the rivers Bosna and Una, in ecologically similar habitats, that is, in relatively clean waters with a small amount of organic material (water quality category I or II). A. pallipes is predominantly present in the Neretva River basin, partly in the Una River basin and in the Cetina River basin, forming both river and lake populations (in the Boracko Lake are present in particularly large numbers) waters with oxygen concentrations greater than $9 \mathrm{mg} \cdot \mathrm{L}^{-1}$ and BOD5 values less than $2 \mathrm{mg} \cdot \mathrm{L}^{-1}$. A. astacus is present in a dense population in waters of the Drina River basin (as river and lake populations), but occurs also in the tributaries of the rivers Bosna (midstream) and Sava. So far, A. leptodactylus was found only at the two localities: Sava River (Brčko area) and Miljacka River (upstream of Sarajevo), in the waters quality of category II (beta-mesosaprobic). Allochthonous species have not been yet recorded in the freshwater habitats of Bosnia and Herzegovina.

\section{RÉSUMÉ}

\section{Les écrevisses d'eau douce en Bosnie-Herzégovine : premières données sur leur distribution}

\section{Mots-clés : écrevisse, diversité, Bosnie- Herzégovine}

Le but de cet article est de synthétiser les données disponibles sur la distribution des écrevisses d'eau douce en Bosnie-Herzégovine, en regroupant les données de la littérature et celles de nos propres investigations. Selon les données historiques, l'existence de quatre espèces indigènes est rapportée sur le territoire de Bosnie-Herzégovine : Astacus astacus (Linnaeus, 1758), A. leptodactylus (Eschscholtz, 1823), Austropotamobius torrentium (Schrank, 1803) et A. pallipes (Lereboullet, 1858). Nos travaux ont confirmé la présence de ces quatre espèces. A. torrentium a été trouvée dans les tributaires des rivières Bosna et Una, dans des habitats semblables, c'est-à-dire dans des eaux claires avec peu de charge

(1) Department of Biology, Faculty of Science Sarajevo, Zmaja od Bosne 33-35, Bosnia and Herzegovina, sadberatb@yahoo.com 
en matière organique (classe de qualité d'eau I ou II). A. pallipes est surtout présente dans le bassin de la rivière Neretva, en partie dans le bassin de la rivière Una, et dans celui de la rivière Cetina, avec des populations en rivière et en lac (particulièrement abondante dans le lac Boracko) aux eaux à des concentrations supérieures à $9 \mathrm{mg} \cdot \mathrm{L}^{-1}$ et des valeurs de BOD5 inférieures à $2 \mathrm{mg} \cdot \mathrm{L}^{-1} \cdot A$. astacus est présente en populations abondantes dans le basin de la rivière Drina (en lac et en rivière), et également dans les tributaires des rivières Bosna (partie médiane) et Sava. Pour l'instant $A$. leptodactylus a été trouvée dans seulement deux localités : rivière Sava (région de Brčko) et Miljacka (amont de Sarajevo), dans des eaux de qualité II (beta-mésosaprobique). Aucune espèce introduite n'a pour l'instant été inventoriée dans les habitats d'eau douce de Bosnie-Herzégovine.

\section{INTRODUCTION}

Bosnia and Herzegovina (B\&H) is located in the central part of the Balkan Peninsula. It is a predominantly hilly and mountainous area with an average altitude of $500 \mathrm{~m}$. The Dinaric mountain chain (in a northwest to southeast direction) makes the relief of Bosnia and Herzegovina with well-developed underground karst forms which classify the territory of Bosnia and Herzegovina as one of the richest holokarst regions in the world. In the past, the Tethys ocean covered a significant portion of the region of Bosnia and Herzegovina, which resulted in the formation of metamorphic rocks in the region of the central Herzegovinian Dinarides. Many springs of rivers and streams are situated in the Dinarides. Isolated karstic fields with their distinctive geological, pedological, hydrological (ground- and underground waters) and other characteristics are of particular interest because of a great diversity of freshwater organisms and a high level of endemic species. Generally, Bosnia and Herzegovina is recognised as an area with a high level of biodiversity and many endemic species, as well as an area with good quality waters. Hydrographically, Bosnia and Herzegovina belongs to both the Black Sea basin (70\%) and the Adriatic Sea basin (30\%). The water bodies of its northern part flow out into the Black Sea basin, and the southern ones into the Adriatic Sea.

The distribution of crayfish in Bosnia and Herzegovina (B\&H), including knowledge about the exact number of all genera and species (indigenous or not) present in the waters of $\mathrm{B} \& \mathrm{H}$, is still insufficiently investigated. The first records of freshwater crayfish in the area of Bosnia and Herzegovina originate from Entz (1914), who stated that Astacus leptodactylus (Eschscholtz, 1823) lives in the Bosna river, and Astacus astacus (Linnaeus, 1758) (as Astacus fluviatilis) lives in the area of Livno. Later, Karaman (1929) stated that the rivers Sava, Bosna, Drina and their tributaries are inhabited by Astacus astacus (as Potamobius fluviatilis fluviatilis and as Potamobius fluviatilis balcanicus), Austropotamobius torrentium (Schrank, 1803) (as Potamobius torrentium), and Astacus leptodactylus. During the 60s, Karaman (1961) recorded the presence of Astacus leptodactylus (as Astacus (Pontastacus) leptodactylus salinus) in the Krivaja river and Austropotamobius pallipes (Lereboullet, 1858) in the region of Herzegovina. Albrecht (1982b) reported the occurrence of Astacus astacus in the Cetina river, whose occurrence was later also confirmed by Šanda and Petrusek (2008). Šanda and Petrusek (2008) found Austropotamobius pallipes in the Bregava River, Fatničko polje, Nevesinjsko polje and Mostarsko Blato.

Until recently, the crayfish populations have never been intensively investigated in Bosnia and Herzegovina, and this is the reason why so little is known about the species and their distribution in this area. However, in the last decade, the water framework directive for water biomonitoring in $\mathrm{B} \& \mathrm{H}$ was established (under the Ministry for Agriculture, Water Management and Forestry, as well as the Agency for the Watershed of the Adriatic Sea and the Water Agency for the Sava River Basin, partly supported by the EU) - this included investigations into freshwater crayfish in $\mathrm{B} \& \mathrm{H}$ and part of these findings from the reports are included in the results of this paper. The main goal of this work is to present recent data on the distribution 
Table I

Saprobic classes (Wegl, 1983).

Tableau I

Classes de saprobie (Wegl, 1983).

\begin{tabular}{|l|c|c|}
\hline Degree of saprobity & Saprobic index (S) & Saprobic category \\
\hline Oligosaprobic & $1.00-1.50$ & I \\
\hline Oligosaprobic to $\boldsymbol{\beta}$-mesosaprobic & $1.51-1.80$ & I-II \\
\hline $\boldsymbol{\beta}$-mesosaprobic & $1.81-2.30$ & II \\
\hline $\boldsymbol{\beta}$ - to $\boldsymbol{\alpha}$-mesosaprobic & $2.31-2.70$ & II-III \\
\hline $\boldsymbol{\alpha}$-mesosaprobic & $2.71-3.20$ & III \\
\hline $\boldsymbol{\alpha}$-mesosaprobic to polysaprobic & $3.21-3.50$ & III-IV \\
\hline Polysaprobic & $3.51-4.00$ & IV \\
\hline
\end{tabular}

of freshwater crayfish in Bosnia and Herzegovina and make comparisons with previously published data (especially with historical data from the beginning of the 20th century), to relate these findings to the water quality in $\mathrm{B} \& \mathrm{H}$ (particular species of crayfish as indicators of water quality) and to compare current distribution with the distribution of Astacidae in the neighbouring countries (native and introduced species).

\section{MATERIALS AND METHODS}

To get a better insight into the distribution of the freshwater crayfish in Bosnia and Herzegovina, we pooled historical data and data from recent observations. Historical data was compiled from the literature (Entz, 1914; Karaman, 1929, 1961, 1962, 1963; Albrecht, 1982b; Trožić-Borovac et al., 2007; Sanda and Petrusek, 2009) and the astacological collection of the National Museum of Bosnia and Herzegovina. Fieldwork to evaluate the current distribution of the crayfish was performed between 2000 and 2010, taking into account various freshwater habitats throughout Bosnia and Herzegovina.

Crayfish were caught by hand or with traps made from PVC bottles (cylindrical shape, with funnel entrances at both ends made from plastic net, mesh size $2 \mathrm{~mm}$ ).

For the localities where crayfish were found (determined and returned to the habitat), GPS coordinates, altitude, and the basic physical and chemical parameters of the water were measured. Water temperature, oxygen concentration, saturation and conductivity measurements were made by the Wissenchaftich - Technische Werkstätten (WTW - multi 340i/SET) terrain set. Biochemical oxygen demand (BOD5) was measured by the Winkler method (standard methods for the examination of water and wastewater 20th edn.) in the Laboratory of Chemistry at the Faculty of Science in Sarajevo.

For most sites, the general level of saprobity, according to the biological parameters, is indicated. Categorisation of the saprobic level and water quality is done on the basis of the obtained results of the saprobic index - see Table I.

The saprobic index (Pantle and Buck, 1955) is measured on the basis of phytobenthos and zoobenthos composition by the formula:

$$
\mathrm{S}=\frac{\sum(h \mathrm{~s})}{\sum h}
$$

where:

$S$ - saprobic index,

$s$ - saprobic value 1.0-4.0 (Wegl, 1983),

$h$ - number of ind. of each taxon.

Saprobic data are the result of research, a part of the biological monitoring which was done by the Faculty of Science for the Agency for the Watershed of the Adriatic Sea and the Water Agency for the Sava River Basin. 


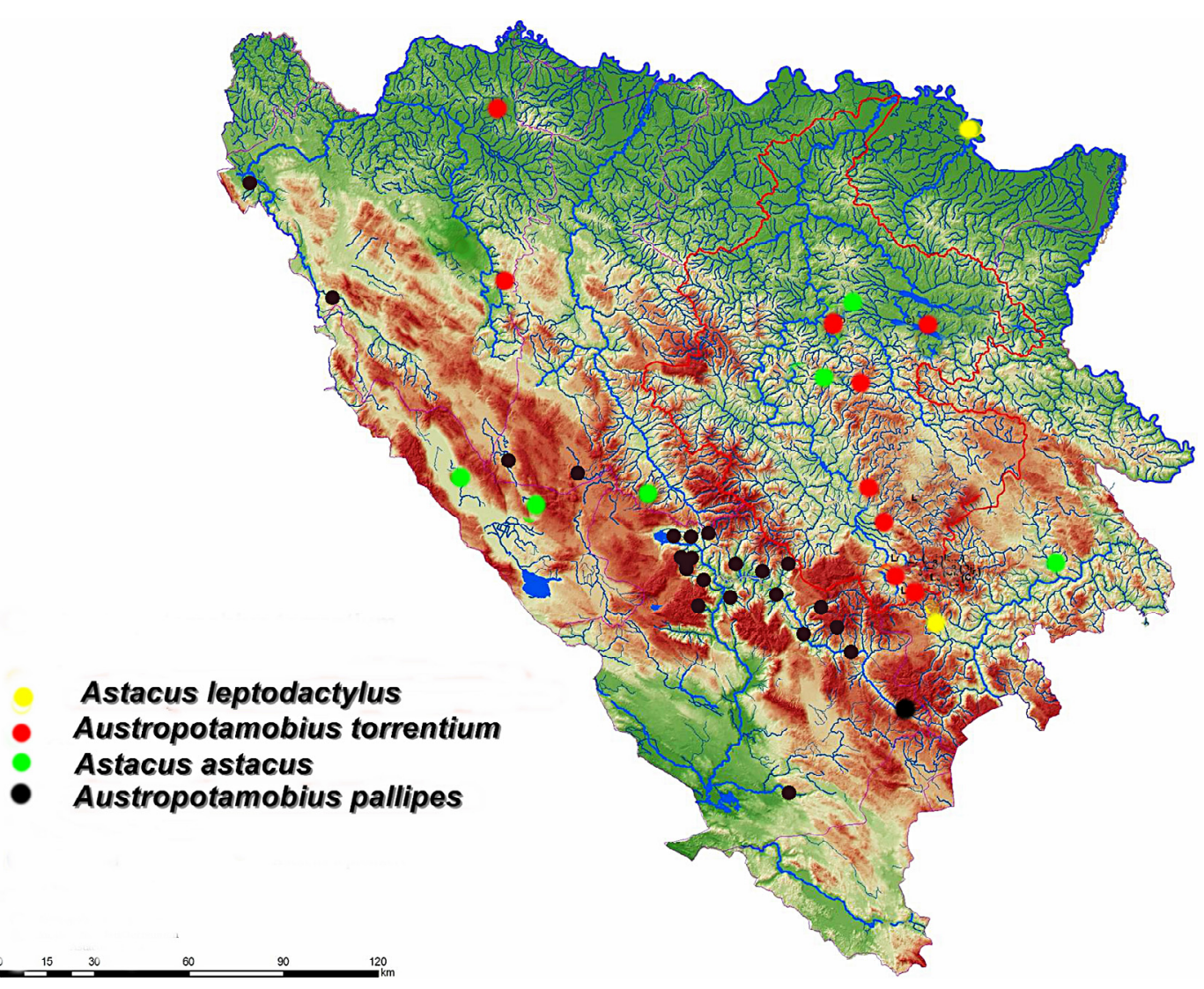

Figure 1

Crayfish distribution from the family of Astacidae.

Figure 1

Distribution des écrevisses de la famille des Astacidés.

Values of total length and weight of individuals of 30 species were measured in the field: Astacus astacus (Praca), Austropotamobius pallipes (Boracko Lake) and A. torrentium (Masicki creek) (Table IV).

The crayfish were determined according to Souty-Grosset et al. (2006).

\section{RESULTS}

According to historical data, the existence of four native European species was reported within the territory of Bosnia and Herzegovina: Astacus astacus (Linnaeus, 1758), A. leptodactylus (Eschscholtz, 1823), Austropotamobius torrentium (Schrank, 1803) and A. pallipes (Lereboullet, 1858) (Table II). According to this data, the tributaries of the Bosna River were inhabited by $A$. leptodactylus and $A$. astacus; the tributaries of the Drina River by $A$. astacus; and the Cetina River by Astacus astacus, $A$. pallipes and $A$. torentium, while the Neretva River was only inhabited by $A$. pallipes. However, according to the crayfish collection from the National Museum of Sarajevo, the presence of only three species was recorded (each species is represented by only one individual in the collection): $A$. leptodactylus (from the Bosna River), A. torrentium (from an unnamed tributary of the Bosna River) and A. astacus (of unknown origin).

Recent research (Figure 1) indicates that the species $A$. astacus was recorded in localities of $180 \mathrm{~m}$ to $1128 \mathrm{~m}$ altitude (Table III), and that the species $A$. leptodactylus was recorded in two localities which are in the water class with present organic matter: $\beta$-mesosaprobic (II). 


\section{Table II}

Historical data on the presence of freshwater crayfish in Bosnia and Herzegovina.

Tableau II

Données historiques de la présence des écrevisses d'eau douce en Bosnie-Herzégovine.

\begin{tabular}{|c|c|c|c|}
\hline Author & Site & Name used for species & Current name of species \\
\hline \multirow{5}{*}{$\begin{array}{l}\text { Entz } \\
(1914)\end{array}$} & Livno & Astacus fluviatilis Linnaeus & $\begin{array}{l}\text { Astacus astacus } \\
\text { (Linnaeus, 1758) }\end{array}$ \\
\hline & Livno & Astacus torrentium (Shrank) & $\begin{array}{c}\text { A. torrentium } \\
\text { (Schrank, 1803) }\end{array}$ \\
\hline & Bosna & Astacus leptodactylus Eschscholtz & $\begin{array}{l}\text { Astacus leptodactylus } \\
\text { Eschscholtz, } 1823\end{array}$ \\
\hline & Donja Tuzla & $\begin{array}{l}\text { Astacus fluviatilis } \\
\text { Linnaeus }\end{array}$ & $\begin{array}{l}\text { Astacus astacus } \\
\text { (Linnaeus, 1758) }\end{array}$ \\
\hline & \begin{tabular}{|c|}
$\begin{array}{c}\text { Outskirts of Visoko } \\
\text { (Bosna) }\end{array}$ \\
\end{tabular} & Astacus fluviatilis Linnaeus & $\begin{array}{l}\text { Astacus astacus } \\
\text { (Linnaeus, 1758) }\end{array}$ \\
\hline \multirow{8}{*}{$\begin{array}{l}\text { Karaman } \\
(1929)\end{array}$} & Sava & $\begin{array}{c}\text { Potamobius fluviatilis fluviatilis } \\
\text { Linnaeus }\end{array}$ & $\begin{array}{l}\text { Astacus astacus } \\
\text { (Linnaeus, 1758) }\end{array}$ \\
\hline & Bosna (Sarajevo) & $\begin{array}{l}\text { Potamobius fluviatilis fluviatilis } \\
\text { Linnaeus }\end{array}$ & $\begin{array}{l}\text { Astacus astacus } \\
\text { (Linnaeus, 1758) }\end{array}$ \\
\hline & Drina & $\begin{array}{c}\text { Potamobius fluviatilis fluviatilis } \\
\text { Linnaeus }\end{array}$ & $\begin{array}{l}\text { Astacus astacus } \\
\text { (Linnaeus, 1758) }\end{array}$ \\
\hline & Cetina & $\begin{array}{c}\text { Potamobius fluviatilis fluviatilis } \\
\text { Linnaeus }\end{array}$ & $\begin{array}{l}\text { Astacus astacus } \\
\text { (Linnaeus, 1758) }\end{array}$ \\
\hline & Cetina & $\begin{array}{c}\text { Potamobius pallipes orientalis } \\
\text { Karaman }\end{array}$ & $\begin{array}{c}\text { Austropotamobius pallipes } \\
\text { (Lereboullet, 1858) }\end{array}$ \\
\hline & Sliv Bosne & $\begin{array}{c}\text { Potamobius torrentium torrentium } \\
\text { (Schrank) }\end{array}$ & $\begin{array}{c}\text { Austropotamobius torrentium } \\
\text { (Schrank, 1803) }\end{array}$ \\
\hline & Cetina & $\begin{array}{c}\begin{array}{c}\text { Potamobius torrentium torrentium } \\
\text { (Schrank) }\end{array} \\
\end{array}$ & $\begin{array}{c}\text { Austropotamobius torrentium } \\
\text { (Schrank, 1803) }\end{array}$ \\
\hline & Sava & $\begin{array}{c}\text { Potamobius leptodactylus } \\
\text { Eschscholtz }\end{array}$ & $\begin{array}{l}\text { Astacus leptodactylus } \\
\text { (Eschscholtz, 1823) }\end{array}$ \\
\hline \multirow{3}{*}{$\begin{array}{l}\text { Karaman } \\
(1961)\end{array}$} & Gacko (Musnica) & $\begin{array}{l}\text { Austropotamobius italicus } \\
\text { ssp. carsicus Karaman }\end{array}$ & $\begin{array}{c}\text { Austropotamobius pallipes } \\
\text { (Lereboullet, 1858) }\end{array}$ \\
\hline & $\begin{array}{l}\text { Herzegovina } \\
\text { (Neretva) }\end{array}$ & $\begin{array}{l}\text { Austropotamobius italicus } \\
\text { ssp. carsicus Karaman }\end{array}$ & $\begin{array}{c}\text { Austropotamobius pallipes } \\
\text { (Lereboullet, 1858) }\end{array}$ \\
\hline & Krivaja & $\begin{array}{c}\text { Astacus (P.) leptodactylus salinus } \\
\text { (Nordmann, 1842) }\end{array}$ & $\begin{array}{c}\text { Astacus leptodactylus } \\
\text { Eschscholtz, } 1823\end{array}$ \\
\hline \begin{tabular}{|l|} 
Albrecht \\
(1982b) \\
\end{tabular} & Cetina & Astacus astacus (Linnaeus) & $\begin{array}{l}\text { Astacus astacus } \\
\text { (Linnaeus, 1758) }\end{array}$ \\
\hline \multirow{7}{*}{$\begin{array}{l}\text { Sanda } \\
\text { and } \\
\text { Petrusek } \\
(2009)\end{array}$} & Bregava & $\begin{array}{c}\begin{array}{c}\text { Austropotamobius pallipes } \\
\text { (Lereboullet, 1858) }\end{array} \\
\end{array}$ & $\begin{array}{c}\text { Austropotamobius pallipes } \\
\text { (Lereboullet, 1858) }\end{array}$ \\
\hline & $\begin{array}{l}\text { Nevesinjsko } \\
\text { field }\end{array}$ & $\begin{array}{l}\text { Austropotamobius pallipes } \\
\text { (Lereboullet, 1858) }\end{array}$ & $\begin{array}{l}\text { Austropotamobius pallipes } \\
\text { (Lereboullet, 1858) }\end{array}$ \\
\hline & $\begin{array}{l}\text { Fatnicko } \\
\text { field }\end{array}$ & $\begin{array}{l}\text { Austropotamobius pallipes } \\
\text { (Lereboullet, 1858) }\end{array}$ & $\begin{array}{l}\text { Austropotamobius pallipes } \\
\text { (Lereboullet, 1858) }\end{array}$ \\
\hline & $\begin{array}{l}\text { Mostarsko } \\
\text { Blato Lake }\end{array}$ & $\begin{array}{l}\text { Austropotamobius pallipes } \\
\text { (Lereboullet, 1858) }\end{array}$ & $\begin{array}{l}\text { Austropotamobius pallipes } \\
\text { (Lereboullet, 1858) }\end{array}$ \\
\hline & $\begin{array}{l}\text { Mostarsko } \\
\text { Blato Lake }\end{array}$ & $\begin{array}{l}\text { Astacus astacus } \\
\text { (Linnaeus, 1758) }\end{array}$ & $\begin{array}{l}\text { Astacus astacus } \\
\text { (Linnaeus, 1758) }\end{array}$ \\
\hline & Milac & $\begin{array}{l}\text { Astacus astacus } \\
\text { (Linnaeus, 1758) }\end{array}$ & $\begin{array}{l}\text { Astacus astacus } \\
\text { (Linnaeus, 1758) }\end{array}$ \\
\hline & Glamoc & $\begin{array}{c}\text { Austropotamobius torrentium } \\
\text { (Schrank, 1803) }\end{array}$ & $\begin{array}{c}\text { Austropotamobius torrentium } \\
\text { (Schrank, 1803) }\end{array}$ \\
\hline
\end{tabular}




\begin{tabular}{|c|c|c|c|c|c|c|c|c|c|}
\hline 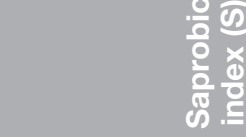 & & 1 & $\stackrel{?}{r}$ & $\stackrel{n}{\infty}$ & $\stackrel{?}{r}$ & 多 & 1 & $\stackrel{N}{N}$ & $\stackrel{\infty}{\infty}$ \\
\hline$\frac{9}{\mathrm{D}}$ & 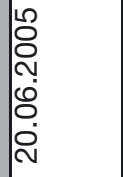 & 1 & 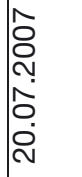 & 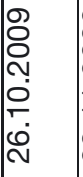 & 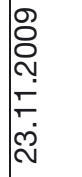 & 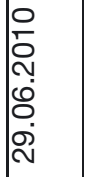 & $\begin{array}{l}0 \\
0 \\
0 \\
0 \\
0 \\
0 \\
\omega \\
N \\
N\end{array}$ & 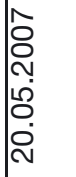 & 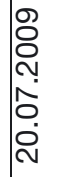 \\
\hline Temp. $\left({ }^{\circ} \mathbf{C}^{-1}\right)$ & 尺 & N & $\nexists$ & $\nexists$ & $m$ & $\dddot{0}$ & $\stackrel{6}{\circ}$ & 10 & $\nexists$ \\
\hline $\mathrm{BPK}_{5}\left(\mathrm{mg} \cdot \mathrm{L}^{-1}\right)$ & $\begin{array}{l}\mathscr{D} \\
\infty \\
\sim\end{array}$ & 1 & 会 & $\mid$ & o & $\begin{array}{l}\infty \\
\infty \\
\infty \\
0\end{array}$ & 1 & $\stackrel{o}{\circ}$ & $\frac{n}{2}$ \\
\hline $\mathrm{O}_{2}\left(\mathrm{mg} \cdot \mathrm{L}^{-1}\right)$ & 잉 & 1 & $\stackrel{\sim}{\dddot{N}}$ & 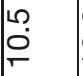 & 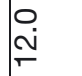 & 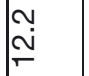 & 1 & $\stackrel{n}{\rightleftarrows}$ & $\stackrel{m}{\leftarrow}$ \\
\hline $\begin{array}{l}\text { Conductivity } \\
\left(\mu \mathrm{S} \cdot \mathrm{cm}^{-2}\right)\end{array}$ & ? & 1 & న & 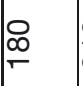 & $\frac{0}{N}$ & î & 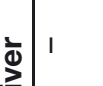 & $\frac{0}{i}$ & 㞔 \\
\hline Elevation (m) & in & 1 & 象 & ৪ & 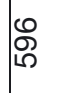 & 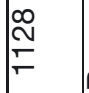 & 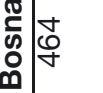 & $\stackrel{શ}{\mp}$ & $\underset{\infty}{\infty}$ \\
\hline 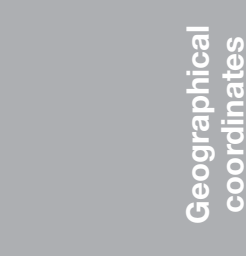 & 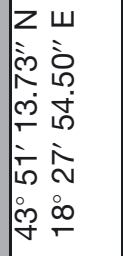 & 1 & 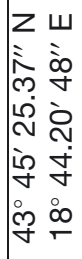 & 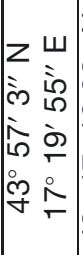 & 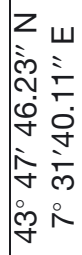 & 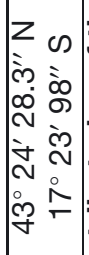 & 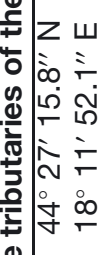 & 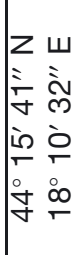 & 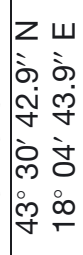 \\
\hline $\begin{array}{l}\frac{\pi}{\circ} \\
\frac{0}{0}\end{array}$ & 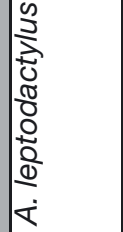 & 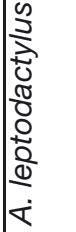 & 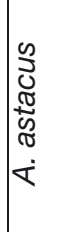 & 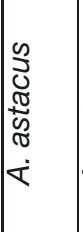 & 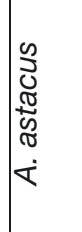 & 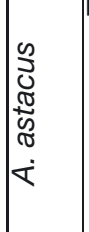 & 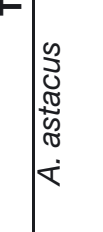 & 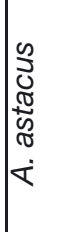 & 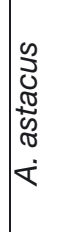 \\
\hline 를 & 10 & N & 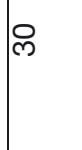 & 10 & 은 & $\sim$ & N & m & a \\
\hline 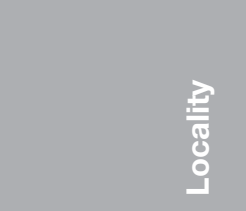 & 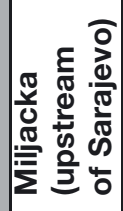 & 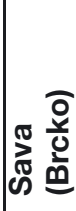 & 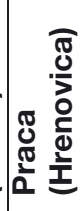 & 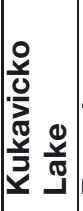 & 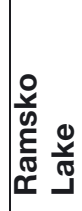 & 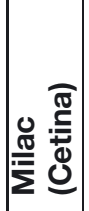 & 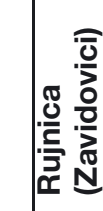 & 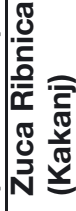 & 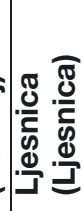 \\
\hline
\end{tabular}




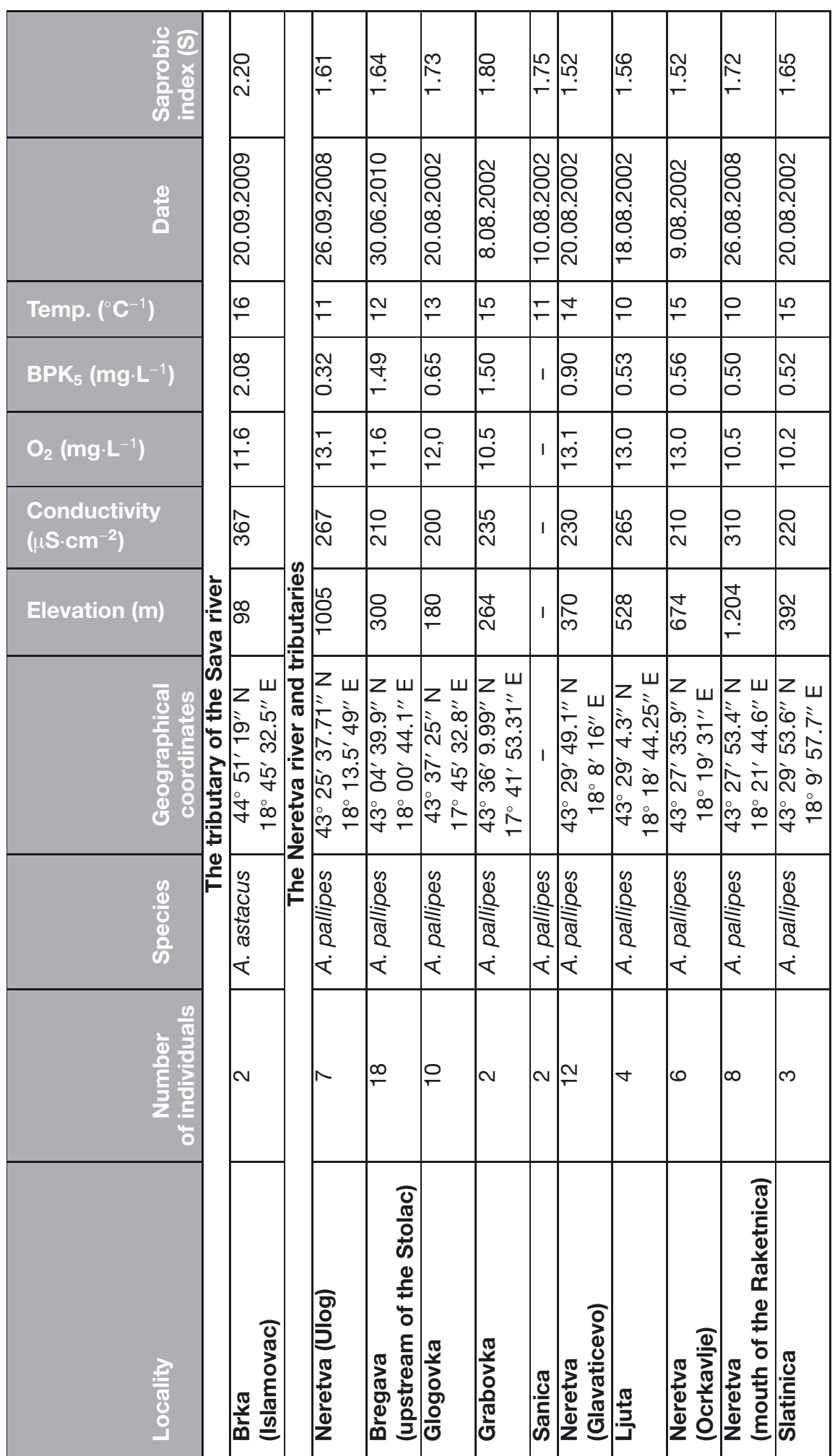

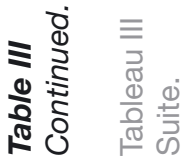




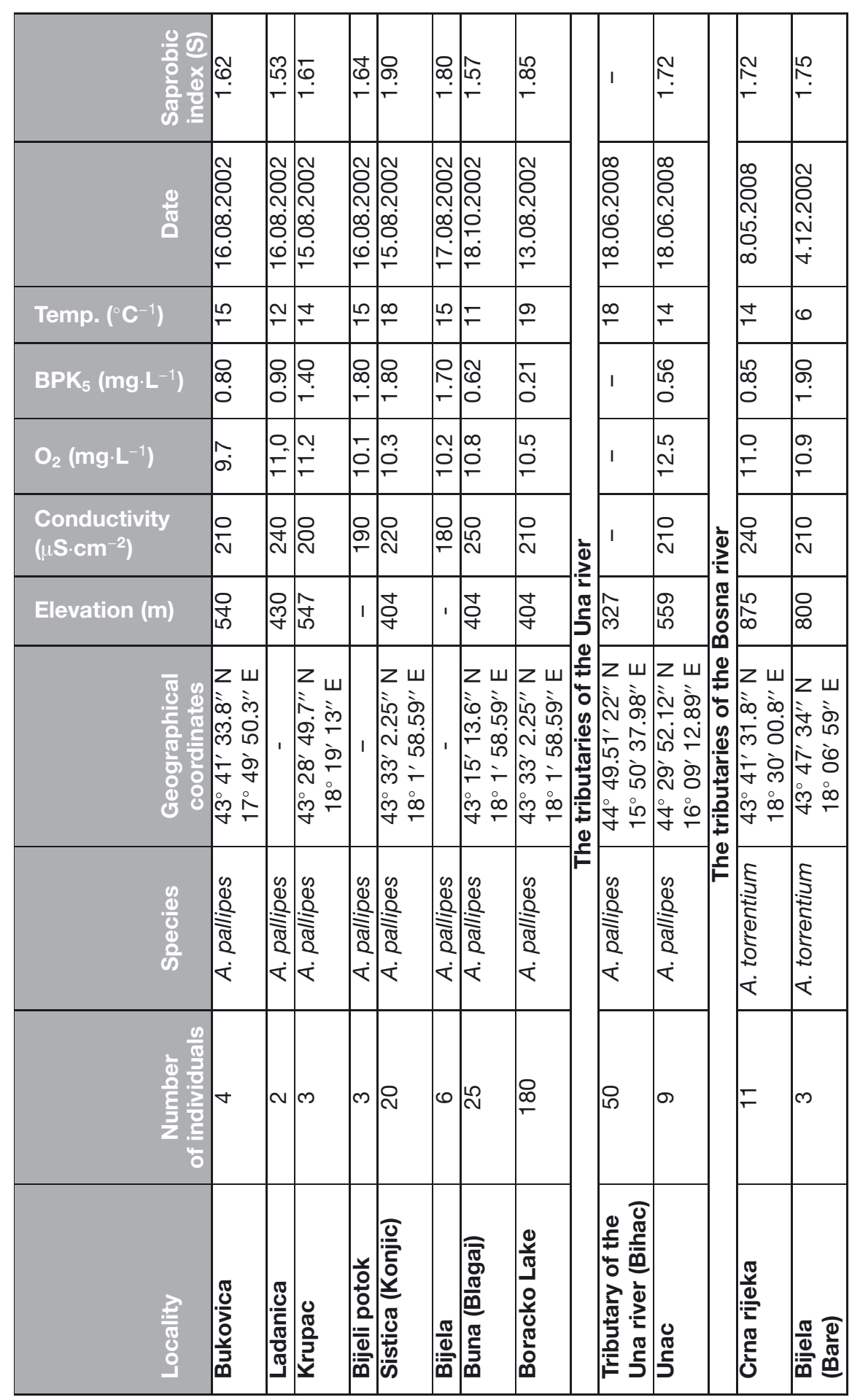




\begin{tabular}{|c|c|c|c|c|c|c|c|c|c|}
\hline 믕 & $\frac{i}{i}$ & مִ & 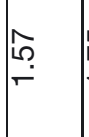 & $\stackrel{n}{n}$ & $\underbrace{n}_{\substack{n \\
n}}$ & $\stackrel{R}{r}$ & 苾 & 1 & 1 \\
\hline$\frac{9}{10}$ & 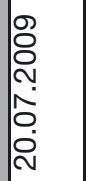 & 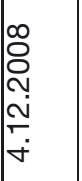 & 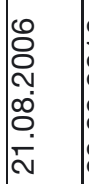 & 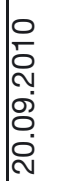 & 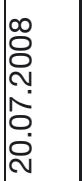 & 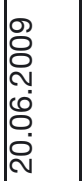 & 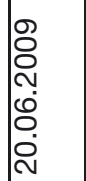 & 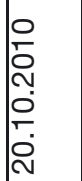 & 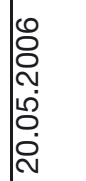 \\
\hline Temp. $\left({ }^{\circ} \mathbf{C}^{-1}\right)$ & $\stackrel{20}{T}$ & $\infty$ & $\mp$ & $\cong$ & $\mp$ & $\mp$ & $ㅇ$ & 1 & $\cong$ \\
\hline $\mathrm{BPK}_{5}\left(\mathrm{mg} \cdot \mathrm{L}^{-1}\right)$ & ְִ. & 垈 & $\begin{array}{l}8 \\
\text { i } \\
\text { i }\end{array}$ & 我 & $\stackrel{0}{\circ}$ & Oי & 安 & 1 & I \\
\hline $\mathrm{O}_{2}\left(\mathrm{mg} \cdot \mathrm{L}^{-1}\right)$ & $\begin{array}{l}\infty \\
\infty\end{array}$ & $\stackrel{\imath}{\check{C}}$ & $\ddot{0}$ & 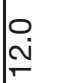 & 我 & क़ & 吕 & 1 & 1 \\
\hline $\begin{array}{l}\text { Conductivity } \\
\left(u S \cdot \mathrm{cm}^{-2}\right)\end{array}$ & প্ণ & $\frac{0}{i}$ & ڤ్ల & $\frac{0}{i}$ & 尺্লে & 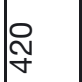 & @ & 1 & 1 \\
\hline Elevation (m) & R & 争 & $\frac{0}{1}$ & 总 & 灾 & i & 冓 & Nิ & 总 \\
\hline 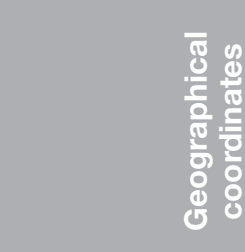 & 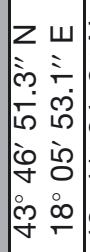 & 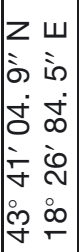 & 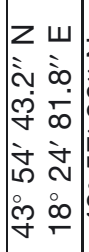 & 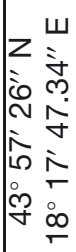 & 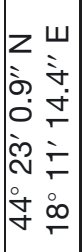 & 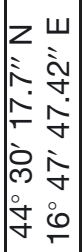 & 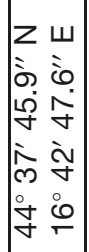 & 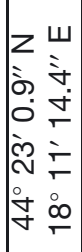 & 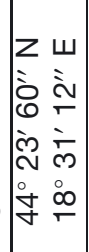 \\
\hline $\begin{array}{l}\frac{8}{0} \\
\frac{\Phi}{0} \\
\frac{8}{\infty}\end{array}$ & 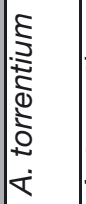 & 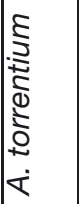 & 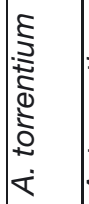 & 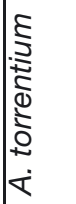 & 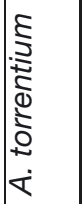 & 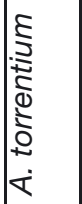 & 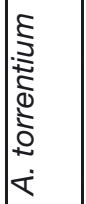 & 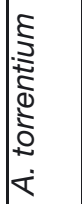 & 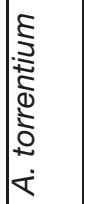 \\
\hline 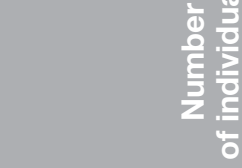 & $\forall$ & $\nabla$ & $F$ & $\stackrel{1}{\Sigma}$ & p p. & $\infty$ & 음 & 0 & $\cong$ \\
\hline 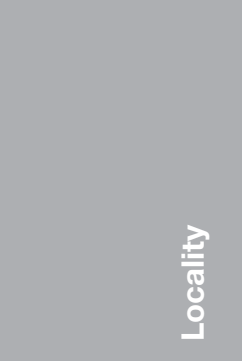 & 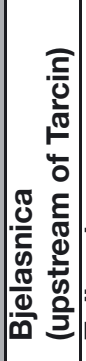 & 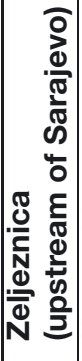 & 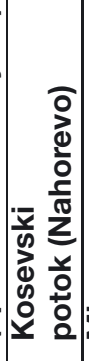 & 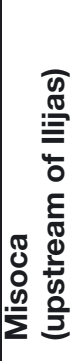 & 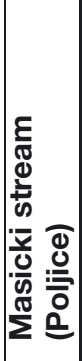 & 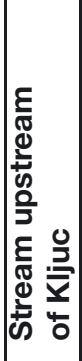 & 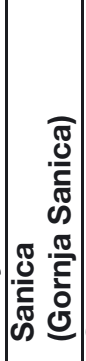 & 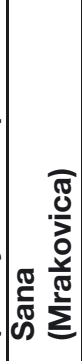 & 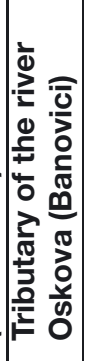 \\
\hline
\end{tabular}

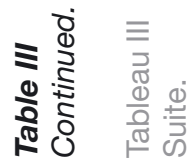


The noble crayfish is tied to waters with slight pollution or oligosaprobic to $\beta$-mesosaprobic (I/II). Taking into consideration that the species has a relatively low saprobic value of 1.2 (Wegl, 1983) and indicator value of 3 in its distribution, it is a good indicator of oligosaprobic or clean waters (category I). In the water of this crayfish habitat, a high value of oxygen and low value of BOD5 is characteristic. The presence of this species is registered in waters with a slightly greater amount of organic matter in the river Brka and river Rujnica in Zavidovici. For absolute confirmation of the presence of this species in indicated waterstreams, more extensive research is required, which is planned to be done in the future.

The white-clawed crayfish $A$. pallipes is registered in a great number of localities in the rivers Cetina, Neretva and Una. In its distribution, this species was recorded at altitudes from $180 \mathrm{~m}$ to $1204 \mathrm{~m}$. It is neccessary to stress its great abundance in Boracko Lake, and in subsequent research the existence of this population was confirmed. Water from all streams where whiteclawed crayfish was determined has high values of dissolved oxygen $\left(>9.7 \mathrm{mg} \cdot \mathrm{L}^{-1}\right)$ and values of BOD5 which are $>2$. The temperature measured in the period of sampling varied in a range from $10.8^{\circ} \mathrm{C}$ to $19^{\circ} \mathrm{C}$.

At the locality of Bregava in subsequent research, females (A. pallipes) with eggs were registered, which points to the successful existence of a relatively big population which is spread over the area of $20 \mathrm{~km}$ of watercourse of the river Bregava up to the outskirts of the town Stoc.

Distribution of the stone crayfish $A$. torrentium is limited to the river Bosna and river Una. The studied streams are in the area of a hilly and mountainous zone from $250 \mathrm{~m}$ to $875 \mathrm{~m}$ altitude. At most sites, relatively small populations were registered, but by far the biggest population was registered in Masicki creek (Gostovic) with 30 individuals analysed. The presence of females with eggs was registered within the population. The water of the locality is characterised by relatively low temperatures which are the result of the time of sampling, and lower values of BOD5, which indicate water with a lower amount of allochthonous organic matter. Saprobic values calculated on the basis of biological parameters of water quality indicate water of category I/II, or relatively clean water with a small amount of organic matter in the watercourse.

Morphometric analysis of the populations of Astacus astacus from the river Praca (Hrenovica), Austropotomobius torrentium from Masicki creek (Gostovic) and $A$. pallipes from Boracko Lake of 30 individuals each was carried out (Table IV). According to the results, the greatest length of noble crayfish was $12.43 \mathrm{~cm}$, and the greatest weight was $87 \mathrm{~g}$. The length of Austropotamobius pallipes varied in a range from $8.5 \mathrm{~cm}$ to $12 \mathrm{~cm}$, and weight from $18.6 \mathrm{~g}$ to $50.9 \mathrm{~g}$. A slightly smaller length, which is characteristic for $A$. torrentium, was measured. The greatest length was $8.9 \mathrm{~cm}$, and the smallest $4.3 \mathrm{~cm}$.

A greater number of males was found in all the analysed samples, which is specially highlighted in the population of $A$. astacus where, in the sample of 30 individuals, only 9 were females.

\section{DISCUSSION}

Historical data (Entz, 1914; Karaman, 1929, 1961, 1963; Albrecht, 1982a; Trožić-Borovac et al., 2007; Šanda and Petrusek, 2009) indicate the existence of four indigenous crayfish species from the family of Astacidae, although most of the data are imprecise and incomplete. According to the recent results, the difference in number of habitats for some species is highlighted, as well as the general ecological conditions of habitats they reside in. Europe is inhabited by five indigenous species of the genera Astacus and Austropotamobius (Souty-Grosset et al., 2006) that are widespread in certain regions and subregions.

In Bosnia and Herzegovina, noble crayfish is distributed in localities with high oxygen concentration and lower levels of saprobity. Its tendency for waters with slight organic pollution is also ascertained in Serbia in the catchment area of the river Morava, with oligosaprobic to $\beta$-metamesosaprobic water (Simić et al., 2008). Habitats in which the presence of this species 


\section{Table IV}

The total length and weight of crayfish species: Astacus astacus in the river Praca (30 individuals), Austropotamobius pallipes in the Boracko Lake (30 individuals), and Austropotamobius torrentium (30 individuals) in the Masicki stream (Gostovic); and ratio of females and males.

\section{Tableau IV}

Longueur totale, poids et sexe ratio de 30 individus des espèces d'écrevisses; Astacus astacus de la rivière Praca, Austropotamobius pallipes dans le lac Boracko, et Austropotamobius torrentium dans la rivière Masicki (Gostovic).

\begin{tabular}{|l|c|c|c|}
\hline \multirow{2}{*}{ Parameters } & Astacus astacus & Austropotamobius torrentium & Austropotamobius pallipes \\
\cline { 2 - 4 } & \multicolumn{3}{|c|}{ Number of individuals } \\
\cline { 2 - 4 } & 30 & 30 & 30 \\
\hline Total length (cm) & & & 12 \\
\hline Max. & 12.43 & 8.9 & 8.5 \\
\hline Min. & 4.61 & 4.3 & 10 \\
\hline Mean & 7.43 & 5.74 & 50.9 \\
\hline Weight (g) & & & 18.6 \\
\hline Max. & 87 & 28 & 22 \\
\hline Min. & 3 & 1 & 13 \\
\hline Mean & 18.4 & 6.51 & 17 \\
\hline Number of females & 9 & 12 & 1 \\
\hline Number of males & 21 & 18 & \\
\hline
\end{tabular}

was determined are partly isolated and are not in close contact, which is affirmed in the areas of the region (Maguire and Gottstein-Matocec, 2004; Simić et al., 2008). The occurrence of this species' introduction into lakes is highlighted, in earlier data, in Busko Lake (Maguire and Gottstein-Matocec, 2004), and in data from 1999 in Ramsko Lake (river Neretva basin), Boracko Lake and in Tuzla.

Although in the countries surrounding Bosnia and Herzegovina (Croatia, Serbia and Montenegro) the species of Turkish crayfish (A. leptodactylus) was determined in a greater number of localities of the river Mura and river Sava basins, according to recent data its existence was determined only in the river Sava and near Sarajevo in Bosnia and Herzegovina. In Serbia, as well as in Croatia, this species exists in the Black Sea basin, and its distribution widens into the Ponto-Caspian region (Simić et al., 2008). Within the wider research on the river Krivaja in the period from 2007 to 2010, the presence of this species was not determined, although it is listed in historical data (Karaman, 1961).

The most widespread species in B\&H with localities at a small distance from each other is the white-clawed crayfish Austropotamobius pallipes which is dominantly determined in the Adriatic basin. It is well adapted to conditions of standing-water and flowing-water ecosystems (Maguire, 2002). A large number of individuals is tied to Boracko lake, and individuals occur not only in the littoral zone, but at relatively great depths (to $5 \mathrm{~m}$ ). According to the present data, the species in Europe occurs up to $1500 \mathrm{~m}$ altitude (Souty-Grosset et al., 2006), and in $\mathrm{B} \& \mathrm{H}$ is determined at $1204 \mathrm{~m}$ altitude with dominant occurrence in upper and middle river streams.

Downstream occurrence of this species is tied to tributaries, while in the river Neretva, it is absent up to the delta in Croatia (Maguire and Gottstein-Matocec, 2004).

The species has proved to be very susceptible to organic pollution (Souty-Grosset et al., 2006). In the river Neretva the wastewaters of the town Stolac impact on its distribution and have caused the species to withdraw upstream. This species, in Austria, has shown a tendency to immigrate into habitats of stone crayfish (Sint et al., 2006) which is, in B\&H, to some extent indicated in the Una basin. It is particularly interesting to note the record of this species in the river Una, which is a part of the Black Sea basin and geographically in direct contact with the catchment area of the river Krka in Croatia. Individuals caught in a numerous population upstream of B\&H Bihac (Toplice) show considerable differences in total length (they are longer) and rostrum shape from the individuals in the river Neretva. This issue will be discussed in a separate study. 
The specifity of the area and ecology of the freshwater species Austropotamobius torrentium has caused its occurrence in small rivers of the Sava basin. According to historical data, the species is recorded in the river Cetina, and according to unconfirmed data it also occurs in the river Neretva. The smallest species within the family of Astacidae in $\mathrm{B} \& \mathrm{H}$ has a small population in most of the watercourses, and only has a greater population size in the area of Misoca (outskirts of Sarajevo) and Masicki creek (upstream of Zavidovici). In both localities, during the period of research (July), mature females (with eggs) were found, which points to successful regeneration of the population. According to data on species ecology (Souty-Grosset et al., 2006), the average length of analysed individuals from Masicki creek $(5.74 \mathrm{~cm})$ corresponds to reproductive maturity. The occurrence of females with eggs in July affirms the period of reproduction to be as in other regions of Europe.

The distribution of the analysed European crayfish species from the family of Astacidae indicated their occurrence in habitats from 180 to $1204 \mathrm{~m}$ altitude. Ecological preferences of species point to differences in tendency for habitats. The fact that there is a small number of habitats of Turkish crayfish indicates the general good condition of the waterstream state, which can be concluded only after more detailed studies.

The non-indigenous species Orconectes limosus (Rafinesque, 1817) and Pacifastacus leniusculus (Dana, 1852) were found in this region, and according to the data their occurrence in $\mathrm{B} \& \mathrm{H}$ is expected accross the area of the river Sava (Maguire and Gottstein-Matocec, 2004; Simić et al., 2008). Despite the loss caused by mortality of crayfish (Sanda and Petrusek, 2009) recorded in some localities, crayfish plague was not found, but its occurrence in European waters and the increasing exploitation of water resources for various purposes (Sint et al., 2002) represent a serious danger for these species.

\section{ACKNOWLEDGEMENTS}

This research was funded by the Ministry of Education and Science, Sarajevo Canton.

\section{REFERENCES}

Albrecht H., 1982a. On the origin of the Mediterranean crayfishes. Quad. Lab. Tecnol. Pesca, Ancona, 2-5, 355-362.

Albrecht H., 1982b. Das System der europäischen Flußkrebse (Decapoda, Astacidae): Vorschlang und Begründung. Mitt. Hamb. Zool. Mus. Inst., 79, 187-210.

Entz G., 1914. Über die Flusskrebse Ungarns. Mathematische und Naturwissenschaftliche Berichte aus Ungarn, 30, 67-127, 4 pls. [1912].

Karaman S., 1929. Die Potamobiiden Jugoslaviens. Glasnik Zemaljskog Muzeja u Bosni i Hercegovini, $41,147-150$.

Karaman M.S., 1961. Slatkovodni rakovi Jugoslavije. Publikacije Strucnog Udruzenja za Unapredenje Slatkovodnog Rribarstva Jugoslavije, 3, 1-33.

Karaman M.S., 1962. Ein Beitrag zur Systematic Astacidae (Decapoda). Crustaceana, 3, 173-191.

Karaman M.S., 1963. Studie der Astacidae (Crustacea, Decapoda) II. Teil. Hydrobiologia, 22, 111-132.

Maguire I., 2002. Porodica Astacidae u sjeverozapadnoj Hrvatskoj, Doktorska disertacija, Prirodoslovnomatematski fakultet Sveučilišta u Zagrebu, 652 p.

Maguire I. and Gottstein-Matocec S., 2004. The distribution pattern of freshwater crayfish in Croatia. Crustaceana, 77, 25-47.

Pantle R. and Buck H., 1955. Die Biologische Uberwaschung der Gewaser und die darstellung der Ergebnisse Gas und Waserfach, 96, 604 p. 
Šanda R. and Petrusek A., 2009. Distribution and diversity of crayfishes in the Adriatic drainage of Bosnia and Herzegovina. In: Kozák P. and Kouba A. (eds.), Abstract Book, Future of Native Crayfish in Europe, Regional European Crayfish Workshop, 7th-10th September 2009, Písek, 50.

Simić V., Petrović A., Rajković M. and Paunović M., 2008. Crayfish of Serbia and Montenegro - The population status and the level of endangerment. Crustaceana 81, 1153-1176.

Sint D., Dalla Via J. and Füreder L., 2006. The genus Austropotamobius in the Ausserfern region (Tyrol, Austria) with an overlap in the distribution of $A$. torrentium an A. pallipes populations. Bull. Fr. Pêche Piscic., 380-381, 1029-1040.

Souty-Grosset C., Holdich D.M., Noël P.Y., Reynolds J.D. and Haffner P., 2006. Atlas of Crayfish in Europe, Muséum national d'Histoire naturelle, Paris, $187 \mathrm{p}$.

Trožić-Borovac S., Deljanin L. and Dautbašić M., 2007. Ecological and biosystematic characteristics of stone crayfish Austropotamobius torrentium (Shrank, 1803) from the Nahorevo brook, Works of the Faculty of Forestry, 1, University of Sarajevo, 39-55.

Wegl R., 1983. Index für die Limnosaprobitat. Wasser und Abwasser, 26, 1-175. 